\title{
Convection in a Very Compressible Fluid: Comparison of Simulations With Experiments
}

\author{
H. Meyer ${ }^{1}$, A. Furukawa ${ }^{2}$, A. Onuki ${ }^{2}$ and A.B. Kogan ${ }^{1, *}$ \\ 1. Department of Physics, Duke University, Durham, NC 27708-0305, USA \\ 2. Department of Physics, Kyoto University, Kyoto 606-8502, Japan . \\ *Present address: Department of Physics, MIT, Cambridge, MA 02139-4307, USA.
}

\begin{abstract}
The time profile $\Delta T(t)$ of the temperature difference, measured across a very compressible fluid layer of supercritical ${ }^{3} \mathrm{He}$ after the start of a heat flow, shows a damped oscillatory behavior before steady state convection is reached. The results for $\Delta T(t)$ obtained from numerical simulations and from laboratory experiments are compared over a temperature range where the compressibility varies by a factor of $\approx 40$. First the steady-state convective heat current $j^{\text {conv }}$ as a function of the Rayleigh number $R a$ is presented, and the agreement is found to be good. Second, the shape of the time profile and two characteristic times in the transient part of $\Delta T(t)$ from simulations and experiments are compared, namely 1 ) $t_{\mathrm{osc}}$, the oscillatory period and 2) $t_{\mathrm{p}}$, the time of the first peak after starting the heat flow. These times, scaled by the diffusive time $\tau_{D}$ versus $R a$, are presented. The agreement is good for $t_{\mathrm{osc}} / \tau_{D}$, where the results collapse on a single curve showing a powerlaw behavior. The simulation hence confirms the universal scaling behavior found experimentally. However for $t_{\mathrm{p}} / \tau_{D}$, where the experimental data also collapse on a single curve, the simulation results show systematic departures from such a behavior. A possible reason for some of the disagreements, both in the time profile and in $t_{\mathrm{p}}$ is discussed. In the Appendix a third characteristic time, $t_{\mathrm{m}}$, between the first peak and the first oscillation minimum is plotted and a comparison between the results of experiments and simulations is made.
\end{abstract}

\section{INTRODUCTION}

In a Raleigh-Bénard (RB) cell, the start of a constant heat flow across the fluid layer produces an initial rise in the temperature difference across this layer, $\Delta T(t)$, with a transient profile determined by the fluid convection dynamics, and which then tends to a steady state value, labeled $\Delta T$. Recently such heat flow experiments were carried out $[1,2]$ on a very compressible fluid in its convective state, supercritical ${ }^{3} \mathrm{He}$, along the critical isochore $\langle\rho\rangle=\rho_{c}$, where the critical temperature is $T_{c}=3.318 \mathrm{~K}$. The fluid layer height in the experiments was $L=0.106 \mathrm{~cm}$ and the diameter of the cylindrical cell was $5.7 \mathrm{~cm}$. For this large aspect ratio, the predicted critical Rayleigh number is $R a_{c}=1708$, as was confirmed by the experiments [1]. Over the reduced temperature range $0.009 \leq \epsilon \equiv\left(T-T_{c}\right) / T_{c} \leq 0.2$ where the experiments were analyzed, the isothermal compressibility increases by a factor of $\approx 40$ as $T_{c}$ is approached. The substantial change in the fluid properties along $\rho_{c}$ is reflected in large changes of the transient profile, where damped oscillations were observed after the first peak of $\Delta T(t)$ for $\epsilon \geq 0.009$.

This paper compares the results of experimental data, and of simulations extending those of refs. [3,4], for the time profile $\Delta T(t)$ in the regime where the fluid approaches steady-state convection. Reference is also made of recently published simulation results by Amiroudine and Zappoli [5]. In the simulations done in refs. [3,4], two new terms are added in the heat conduction equation; the first takes into account the adiabatic heating taking place throughout the cell (the "piston effect"), and the second accounts for the adiabatic temperature gradient effect within plumes leading to the Schwarzschild criterion of the convection onset in compressible fluids [6]. In the work of ref. [5] the Navier-Stokes (NS) equation, together with an enthalpy equation were used, without a specific term to simulate the piston effect, since the latter is implicitly taken into account in the work of the pressure forces term in the enthalpy equation. The simulations were made in two dimensions, where the fluid was contained in a cell with the same height $L$ as in the experiment. The aspect ratio was 4 in refs. [3,4] and 2 in ref. [5] with the periodic boundary condition in the horizontal direction in order to reproduce the conditions of the experimental cell with a large aspect ratio. The simulations for $\epsilon=0.05$ in ref [4] extended until $\left[R a-R a_{c}\right] \approx 4 \times 10^{6}$ and until $\approx 1.7 \times 10^{5}$ for other values of $\epsilon$. Simulation results in this paper, except those of $\epsilon=0.05$ in refs. [3,4], are newly obtained using the scheme in ref. [4].

The outline for the remainder of this paper is as follows: First, a general discussion of the profiles $\Delta T(t)$ will be made, with presentation of some examples and an appraisal of the degree of agreement between experiments and simulations. Second, a comparison of the steady state results from experiments and simulations will be presented, expressed in terms of the convection heat current $j^{\text {conv }}$ versus the reduced Rayleigh number $r a^{*} \equiv\left[R a-R a_{c}\right] / R a_{c}$. Third, a comparison of the transients from experiments and simulations will be made by the examination of two "characteristic times", the time $t_{\mathrm{p}}$ of the first peak after the start of the heat current, and $t_{\mathrm{osc}}$, the oscillation 
period in the damped oscillatory decay. Both will be presented in a scaled form, again versus $\left[R a-R a_{c}\right]$. This quantitative comparison illustrates well the agreements and deviations between simulations and experiments, which will be discussed in the Conclusions. In the Appendix, a third characteristic time, $t_{\mathrm{m}}$, between the first peak and the first minimum in the $\Delta T(t)$ profile is shown for both the experiments and the simulations.

\section{GENERAL OBSERVATIONS ON THE PROFILES $\Delta T$ VERSUS TIME.}

We briefly emphasize, as was done in refs $[1,2]$, that the experiments were done under conditions where the stratification from gravity was small and where the temperature changes across the fluid were kept small enough that the changes in the fluid properties across the fluid layer were smaller than a few percent. Thus the conditions for the approximations in a Boussinesq-like fluid in the momentum equation were maintained. In Fig. 9 in ref. [1] a representative evolution is shown of the observed profile $\Delta T(t)$ at $\epsilon=0.05$ as a function of the heat current $q$. Both experiments and simulations show over a certain range of heat flow $Q$ and of temperature a damped oscillatory profile $\Delta T(t)$ as shown in Figs. 1 and 2 of ref. [3], Figs. 1 of ref. [4], both at $\epsilon=0.05$ and Fig.2 of ref. [5] at $\epsilon=0.01$, which are not reproduced here. Considering that the experimental data have not been corrected for the time lag introduced by the temperature recording instrumentation with a time constant of $\tau=1.3 \mathrm{~s}$., the qualitative agreement is quite satisfactory. However as $\epsilon$ increases and the compressibility decreases, the disagreement between the transient regime of experiments and simulations becomes sizeable. This can be seen in Fig.1 at $\epsilon=0.2$ for two values of the heat flow $q \approx 3.7 \times 10^{-7}$ and $2.1 \times 10^{-7} \mathrm{~W} / \mathrm{cm}^{2}$. By contrast the steady-state value for
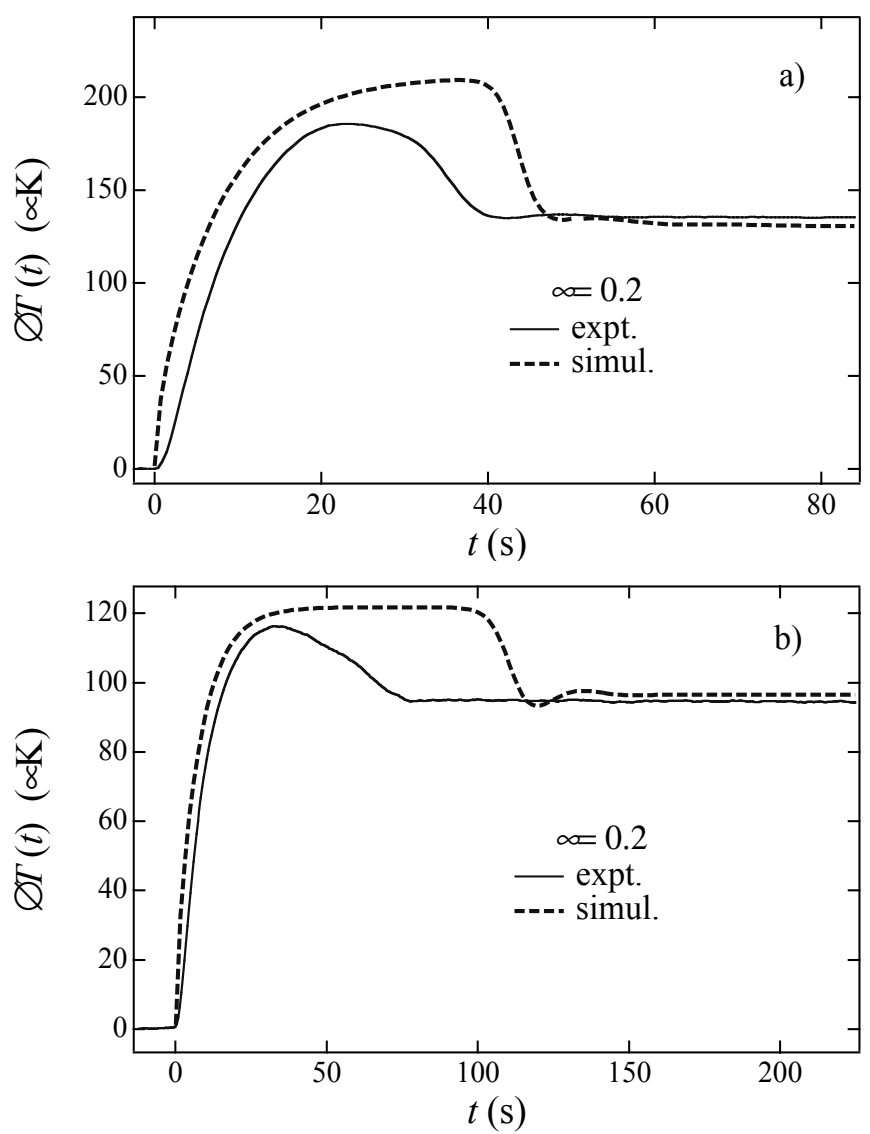

FIG. 1. Plots of the profile $\Delta T(t)$ versus time for two values of the heat flow $q$ (in $\left.10^{-7} \mathrm{~W} / \mathrm{cm}^{2}\right)$ at $\epsilon=0.2$ and comparison between experiments and simulations a) $q=3.89$ (expt), 3.67 (simul.) and b) $q=2.16$ (expt), 2.10 (simul.).

$\Delta T$ reached in both the experiments and in simulations for the same value of $q$ remains closely the same. In the experimental trace for the lower value of $q$, no damped oscillations are seen, but rather a non-exponential decay of the overshoot. This is the regime labeled "truncated oscillations", described in Fig.2 of ref. [2]. (See also Appendix A). Simulations, however, always show damped oscillations in the convective state. 


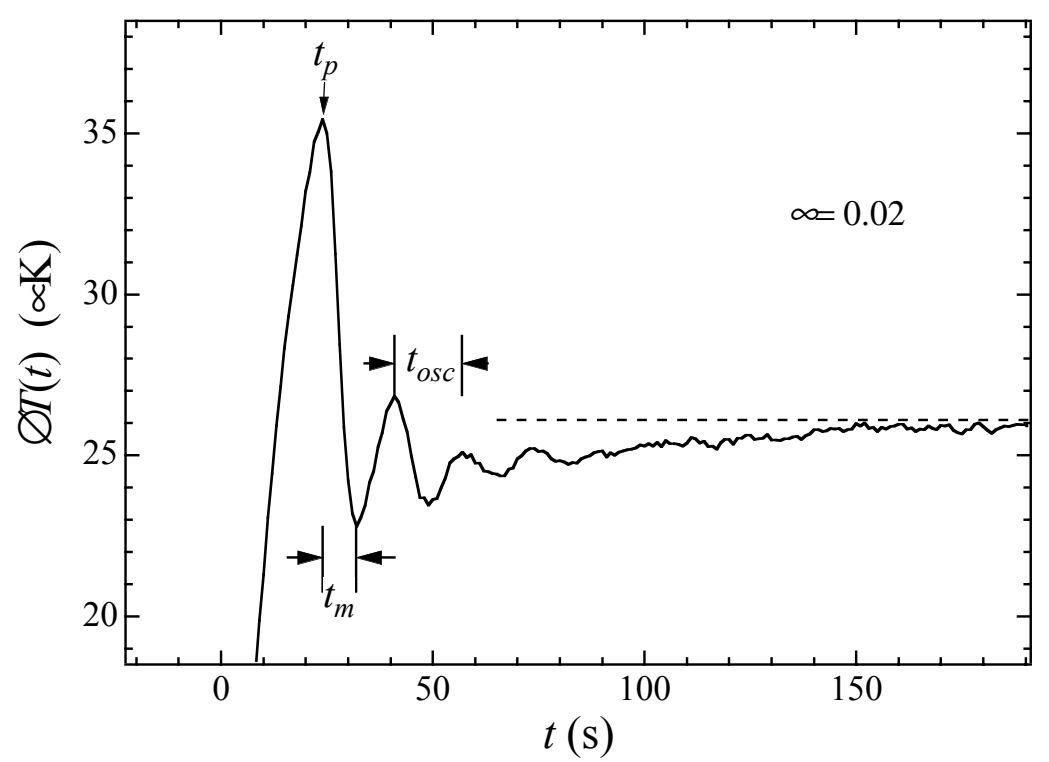

FIG. 2. A representative profile $\Delta T(t)$ after starting the heat current, with the definition of the times $t_{\mathrm{p}}, t_{\mathrm{m}}$ and $t_{\mathrm{osc}}$ and the slow exponential relaxation to the steady-state value of $\Delta T$ (dashed line) with a time constant $\tau_{\text {tail }}$. The recording is for $\epsilon$ $=0.02$ with the heat flow $\left.q=1.6910^{-7} \mathrm{~W} / \mathrm{cm}^{2}\right)$.

In Fig.2, we present an enlarged segment near the peak of $\Delta T(t)$ for a representative trace at $\epsilon=0.02$, which exhibits and defines various characteristic times in the transient. We will consider two of these times in the main part of this paper, namely 1) the peak $t_{\mathrm{p}}$ of the first peak after the start of the heat current and 2) the period $t_{\mathrm{osc}}$ of the damped oscillations.

Furthermore there is $t_{\mathrm{m}}$, the interval between the first peak and the first minimum in the regime of damped oscillations or between the peak and the "kink" to the flat portion in the regime of "truncated oscillations". The discussion of $t_{\mathrm{m}}$ is deferred to Appendix $\mathrm{B}$, because it is more complex than for $t_{\mathrm{osc}}$ and $t_{\mathrm{p}}$. Finally, the profile tends asymptotically from below to the steady state value $\Delta T$ with a relaxation time $\tau_{\text {tail }}$, obtained by fitting this transient tail with a simple exponential, and we note that this feature has not been detected in the simulations either in ref. [4] or in ref. [5].

The transient shape, principally at short times where the changes in $\Delta T(t)$ are rapid, is affected by the instrumentation time constant, and also the modulated minimum shown in Fig.2 makes an analysis of the oscillation amplitude decay rate uncertain. However inspection of the many recorded experimental traces shows that for a given $\epsilon$ (or compressibility), the rates for both the oscillations and their amplitude decay increase with the heat current. For values large enough of $q$ at a given $\epsilon$, the first peak and the oscillations become attenuated and averaged out. (See Fig. 4 of ref [2]). The likely cause for this observation is that $t_{\mathrm{osc}}$ becomes comparable or smaller than the instrumentation time constant. An averaging effect of the oscillations due to a negative interference between non-synchronous plumes that results from the large lateral dimension of the experimental cell was suggested in ref. [4]. This suggestion might be very relevant in the regime $\epsilon<0.009$ where no oscillations could be observed, even when their expected period was well above that of the instrumental time constant (See ref. [2], Section III).

\section{COMPARISON OF STEADY-STATE RESULTS IN EXPERIMENTS AND SIMULATIONS}

In ref. [1] the steady-state experimental results were presented in terms of the dimensionless convective heat current $j^{\text {conv }}$ versus $r a^{*}$. Here $j^{\text {conv }}$ is the ratio of the convective portion of the heat current to that conducted through the fluid at the transition to convection, which leads to the relation [7]

$$
j^{\mathrm{conv}} \equiv(N u-1)\left(r a^{*}+1\right)
$$

where $N u$ is the Nusselt number. Along the critical isochore of a fluid, where the compressibility diverges as $T_{c}$ is approached, Gitterman and Steinberg [8] have shown that for the fluid onset of mechanical instability there is a crossover from the Rayleigh - to the Schwarzschild (or "adiabatic temperature gradient") criterion as $T_{c}$ is approached. Then it has been shown ( [9] and references therein) that at the onset of convection, the temperature drop across the fluid layer is given by 


$$
\Delta T_{\text {onset }}=\Delta T_{R}+\Delta T_{a d}
$$

where $\Delta T_{R}=R a_{c} \times \nu D_{T} / \alpha_{P} g L^{3}$, the familiar Rayleigh term, and $\Delta T_{a d}=L g T \alpha_{P} / C_{P}$, the adiabatic temperature difference contribution (for $\Delta T_{R}$ and $\Delta T_{a d}$ see for instance Tritton's monograph [10]). Here $\nu$ is the kinematic viscosity, $D_{T}$ the thermal diffusivity, $\alpha_{P}$ the isobaric thermal expansion coefficient, $g$ the gravity accelation, $L$ the height of the fluid layer and $C_{P}$ the specific heat at constant pressure.

As has been described in some detail in refs. [1,2], $N u$ and $R a$ have to be corrected for the contribution from the adiabatic temperature gradient. One then obtains

$$
R a_{\text {corr }}=\frac{R a\left(\Delta T-\Delta T_{a d}\right)}{\Delta T} \text { and } N u_{\text {corr }}=\frac{\left(\Delta T_{\text {diff }}-\Delta T_{a d}\right)}{\left(\Delta T-\Delta T_{a d}\right)}
$$

and hence

$$
r a_{\mathrm{corr}}^{*} \equiv\left(R a_{\mathrm{corr}} / R a_{c}-1\right) \quad \text { and } j_{\mathrm{corr}}^{\mathrm{conv}} \equiv\left(N u_{\mathrm{corr}}-1\right)\left(r a_{\mathrm{corr}}^{*}+1\right)
$$

Here $\Delta T_{\text {diff }}$ is the temperature drop across the fluid in the diffusive regime for the same heat current producing the observed $\Delta T$. Because both $j_{\text {corr }}^{\text {conv }}$ and $r a_{\text {corr }}^{*}$ vary over about five orders of magnitude for the range covered by the data, a more sensitive way is to present the ratio $j_{\text {corr }}^{\text {conv }} / r a_{\text {corr }}^{*}$ versus $r a_{\text {corr }}^{*}$. Furukawa and Onuki [4] theoretically justified the validity of the scaling relations in terms of these corrected quantities. This is done in Fig.3a where the points recorded close to the transition to convection and showing rounding instead of a sharp convection onset, have been omitted. Furthermore the data for $\epsilon<0.009$, where no damped oscillations were obtained in the transients, and for which no simulations were carried out, have not been used in this figure. As can be seen, within the scatter all the data points nearly collapse on a common curve. For $r a_{\text {corr }}^{*}<1$, the data extrapolate to a horizontal line with an amplitude of $1.3 \pm 0.1$. This asymptotic result, $j_{\mathrm{corr}}^{\text {conv }}=1.3 \mathrm{r} \mathrm{a}_{\mathrm{corr}}^{*}$, which represents data slightly above the onset of convection, has been discussed in ref. [1] where it had been concluded that the amplitude is consistent with a straight roll convection prediction [11]. In Fig. 3b, the experimental data are replaced by a solid curve representing its average, and the results from simulations are shown by the symbols at various values of $\epsilon$. In the intermediate range of $r a_{\text {corr }}^{*}$ these data points collapse on a curve slightly above the experimental average, and also within their scatter they tend to the same limiting amplitude of 1.3, as do the experiments. Therefore they also imply straight roll convection, a picture which is plausible in the $2 \mathrm{D}$ simulation which represents a cross-section of rolls in a geometry with parallel vertical periodic boundaries.
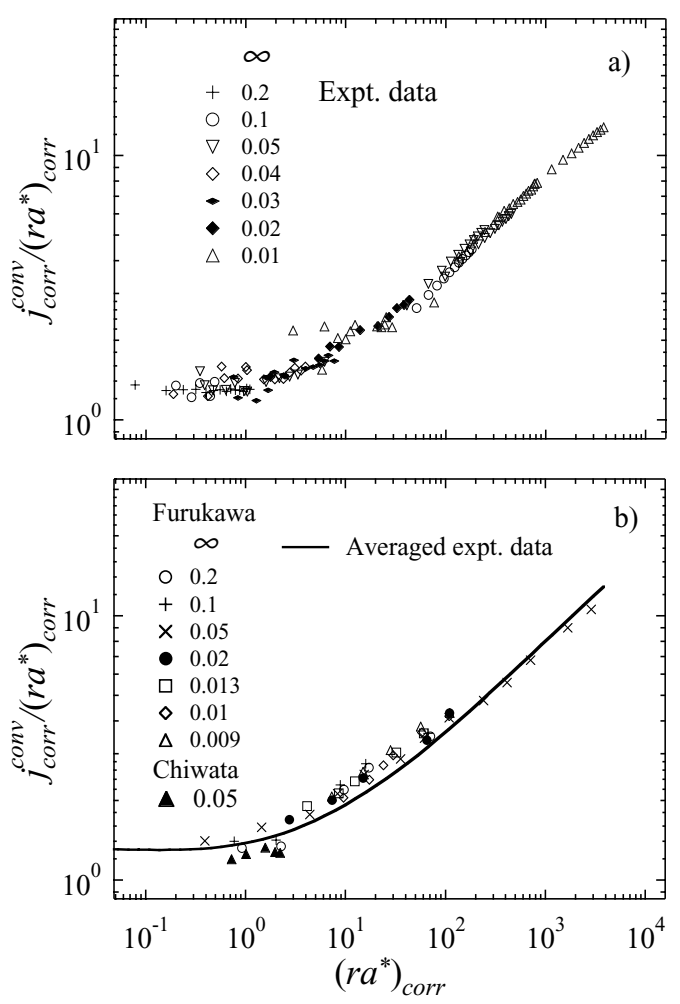


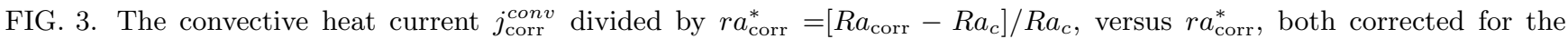
adiabatic temperature gradient contribution. a) Experimental data at various values of $\epsilon$. b) Data from simulations at different values of $\epsilon$ shown by symbols. The solid line is the average of the experimental data in a).

\section{TRANSIENT CHARACTERISTIC TIMES IN EXPERIMENTS AND SIMULATIONS}

\section{A. Background information}

The mechanism responsible for the damped oscillations for a highly compressible fluid at constant average density has been discussed most recently by Furukawa and Onuki [4] and by Amiroudine and Zappoli [5]. From their simulations, these authors presented a detailed analysis of the time evolution of the temperature in various locations in the fluid layer. We refer to their description on how maxima and minima of $\Delta T(t)$ are produced by the "piston effect" that leads to the vertical flow of successive "warm" and "cold" fluid masses. The snapshots of the simulations by Chiwata and Onuki [3] had already pictured the formation of plumes during these processes, and the evidence of warm and cold vertical flows.

In the analysis of the experimental data [2], two characteristic times describing the remarkable oscillatory behavior, $t_{m}$ and $t_{\mathrm{osc}}$, were discussed, which are shown in Fig. 2 for a representative $\Delta T(t)$ profile. The experimental results for the time $t_{\mathrm{p}}$ have not been analyzed before and are presented here for the first time. In order to keep the analysis of the results tractable, their discussion will not include $t_{m}$, which will be dealt with in Appendix A. In the experimental data analysis, the relaxation time $\tau_{\text {tail }}$ to the steady state convection was also presented in ref. [2]. All the data of $t_{\text {osc }}$ and $\tau_{\text {tail }}$ at the various reduced temperatures $\epsilon$, scaled by the diffusion time $t_{D} \equiv L^{2} / 4 D_{T}$, and plotted versus the Rayleigh number difference $\left[R a_{\text {corr }}-R a_{c}\right]$ were found to collapse within a scatter of $\pm 15 \%$ on two respective curves, an unexpected result.

\begin{tabular}{|r|c|c|c|}
\hline$\epsilon$ & $C_{p} / C_{v} \equiv \gamma$ & $B(\gamma) \equiv \tau_{D} / t_{D}$ & $\tau_{D}(\mathrm{~s})$. \\
\hline \hline 0.01 & 119 & 1.01 & 265 \\
0.02 & 57.7 & 1.03 & 134 \\
0.03 & 38.1 & 1.06 & 92.0 \\
0.04 & 28.5 & 1.08 & 69.9 \\
0.05 & 22.8 & 1.10 & 57.0 \\
0.07 & 16.5 & 1.13 & 42.2 \\
0.10 & 11.8 & 1.19 & 31.8 \\
0.20 & 6.48 & 1.35 & 19.8 \\
\hline
\end{tabular}

Table 1. The ratio $C_{p} / C_{v} \equiv \gamma$ for ${ }^{3}$ He along its critical isochore for several values of the reduced temperature $\epsilon$, the ratio $B(\gamma)$ of the diffusive times, calculated from Fig.2 ref.12 (Behringer et al.) and the diffusive time $\tau_{D}$.

We now discuss the choice of the scaling time in the data for $t_{\mathrm{osc}}$ and $t_{\mathrm{p}}$ versus the Rayleigh number. As mentioned above, the diffusion time $t_{D}$ was used as the scale for $t_{\mathrm{osc}}$ in the plot versus $\left[R a_{\text {corr }}-R a_{c}\right]$. This time $t_{D}$ was first used in the Navier Stokes equation leading to the simulations of ref. [3] under the conditions of constant average density and valid for $C_{p} / C_{v} \equiv \gamma>>1$. In the absence of convection, $t_{D}$ can be related to the relaxation time $\tau_{0}=L^{2} / \pi^{2} D_{T}$ of the lowest diffusion mode in a RB - or in a standard thermal conductivity cell [12]. However over the experimental and simulation range where $0.2 \geq \epsilon \geq 0.009$, the condition $\gamma \gg 1$ is only progressively realised as $\epsilon$ decreases to 0.01. In ref. [12] expressions for the solution of $\tau_{0}$ as a function of $\gamma$ were derived. As $\gamma \rightarrow 1, \tau_{0} \rightarrow 4 L^{2} / \pi^{2} D_{T}$, and this last value is the same as for a fluid relaxing at constant pressure. In general $\tau_{0}(\gamma)=B(\gamma) L^{2} / \pi^{2} D_{T}$, where $B(\gamma)=\left(\pi / q_{0} L\right)^{2}$, obtained from Fig.2 of ref [12] with $q_{0}$ the wave number of the lowest mode. $B(\gamma)$ is presented in Table 1 for several values of $\epsilon$ relevant to the experiments and simulations in this paper. As can be seen in this Table, the ratio $\tau_{0}(\gamma) / \tau_{0}(\gamma=\infty)$ decreases with $\epsilon$ and tends to 1 for $\epsilon<0.01$. The use of the time $\tau_{D} \equiv \tau_{0}(\gamma) \pi^{2} / 4$ as a scale for the convection transient characteristic times is therefore preferable to $t_{D}$. It turns out that the choice of $\tau_{D}$ as the scaling time improves the collapsing of the experimental data points for both $t_{\mathrm{osc}}$ and $t_{\mathrm{p}}$. In the following subsections we present and compare these times obtained experimentally and from simulations.

\section{B. the oscillatory period $t_{\mathrm{osc}}$}

In ref. [2], Fig.4 presented $t_{\mathrm{osc}}$ scaled by $t_{D}$ and plotted versus $\left[R a_{\text {corr }}-R a_{c}\right]$. The substitution of $\tau_{D}$ as a scale gives a better collapsing of the data points than does $t_{D}$, particularly at the lower values of $\left[R a_{\text {corr }}-R a_{c}\right]$ where most of the data points at the higher values of $\epsilon$ lie. Within the scatter of $\pm 15 \%$ the data for $10^{3}<\left[R a_{\text {corr }}-R a_{c}\right]<5 \times 10^{5}$ 
can be represented by a power law with an exponent of $-0.52 \pm 0.02$. In Fig. $4 \mathrm{a}$ we present these data and in Fig.4b its average, obtained by a fit to a power law with a corrective term for the larger $R a$ values. Also in Fig. $4 \mathrm{~b}$ we show by symbols the results from the simulations by the Kyoto group (two of the present authors, A.F. and A.O. and Chiwata) and by Amiroudine and Zappoli [5] at various values of $\epsilon$. The agreement appears to be good, though the simulations indicate some small systematic deviations from collapse on a single "universal" line. The basic reason for the apparent power law with an exponent of $-1 / 2$ remains to be understood.
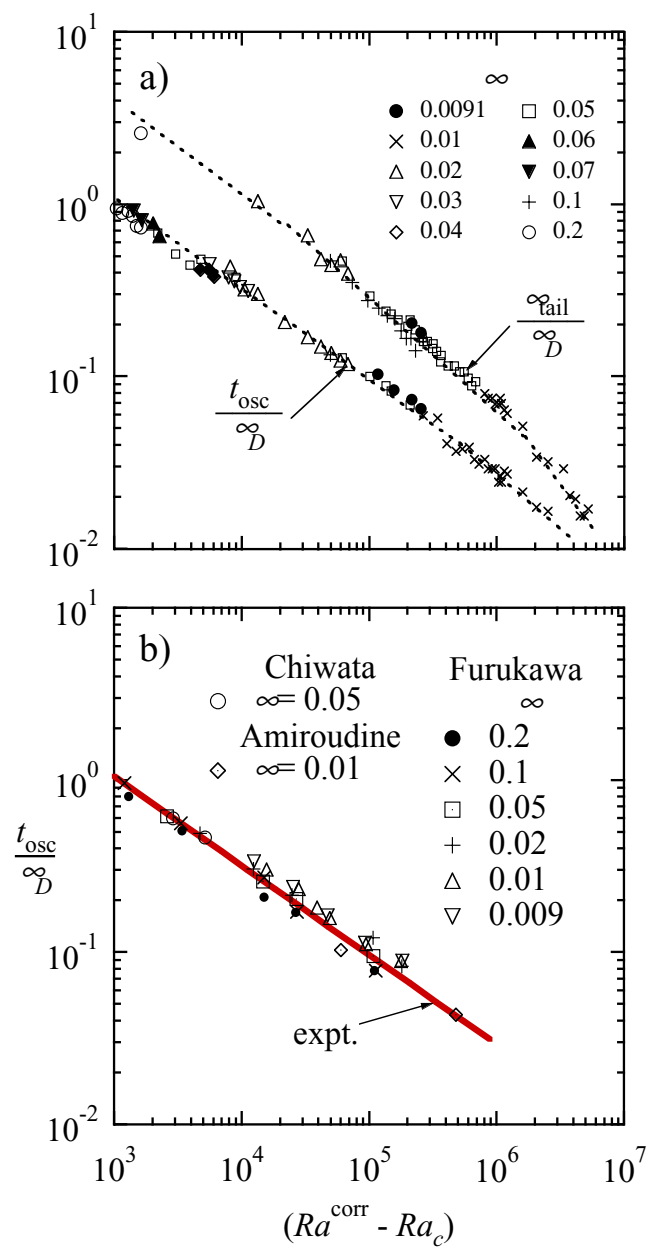

FIG. 4. The oscillation period $t_{\mathrm{osc}}$ and the time $\tau_{\text {tail }}$ for the relaxation to the steady-state, both scaled by the diffusion time $\tau_{D}$, versus $\left[R a^{\text {corr }}-R a_{c}\right]$. a): Experimental data at various values of $\epsilon$. b): Symbols show the simulation data at different values of $\epsilon$, and the solid line is the average of the experimental data in a).

\section{The location of the first peak at $t_{\mathrm{p}}$}

In the experiments, the initial rise of the measured transient $\Delta T(t)$ after the start of the heat flow across the fluid layer is affected by the time constant of the thermometer circuitry, $\tau=1.3 \mathrm{~s}$. as described in ref. [1]. This is especially so at short times, when the $\Delta T(t)$ increases rapidly, and as a result the measured value of the time at the first peak has to be corrected. This was done by comparing the shift in time between the calculated rise of $\Delta T(t)$ in the conducting regime (Eq.3.3 of ref. [4]) and the recorded curve for several values of $\epsilon$ and $q$. This shift was between 2 and $3 \mathrm{~s}$. and $t_{\mathrm{p}}$ was obtained after a crude correction was made by subtracting $\delta t=2 \mathrm{~s}$. from the measured time at the peak. The times $t_{\mathrm{p}}$ used in this analysis ranged from $\approx 100$ to $\approx 7 \mathrm{~s}$. Fig. 5a shows the scaled representation $t_{\mathrm{p}} / \tau_{D}$ of the experimental times versus $\left[R a_{\text {corr }}-R a_{c}\right]$. There is excellent collapse of the data for all the values of $\epsilon$, even extending to the region of $\epsilon<0.009$ where no damped oscillations are observed [2]. In Fig.5b the solid curve represents the average of the experimental data, as obtained by a polynomial fit. Symbols show the results from simulations at various values of $\epsilon$. The data from ref. [3] at $\epsilon=0.05$ 

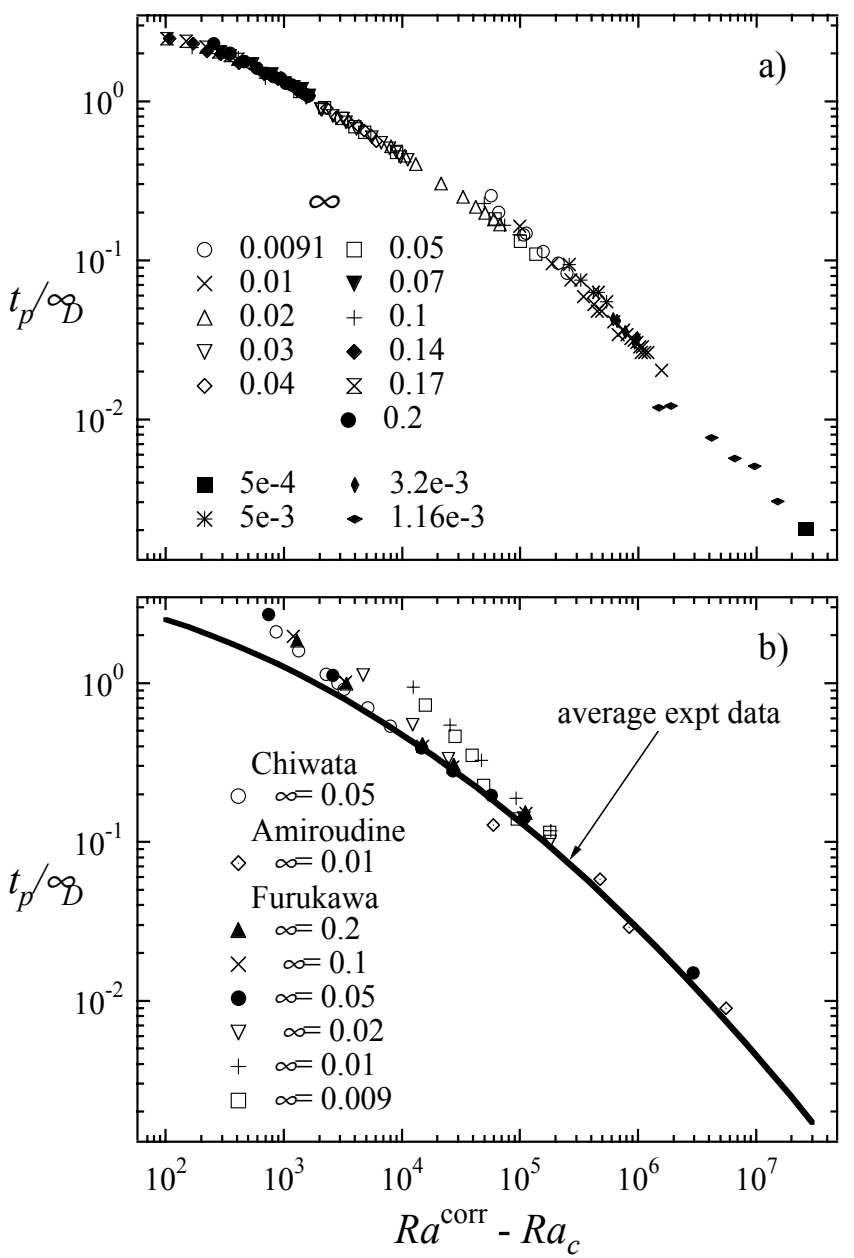

FIG. 5. The time of the first peak $t_{\mathrm{p}}$, scaled by the diffusion time $\tau_{D}$, versus $\left[R a_{\text {corr }}-R a_{c}\right]$. a): The experimental data, where the symbols denote the various values of $\epsilon$ at which the experiments were carried out. b): The curve representing the average of the experimental data from 5 a), compared with the results from simulations, shown as symbols at the various values of $\epsilon$.

and those in the present research are found to be internally consistent. However, contrary to the experimental results, the simulation data do not collapse on a single curve. This discrepancy is expected from the profiles $\Delta T(t)$ shown in fig. 1 where the peak in the simulations lies at substantially longer times $t_{\mathrm{p}}$ than for the experiments. A possible source for the discrepancy between simulations and experiments will be considered below.

\section{DISCUSSION}

First we present general comments on transient observations in Rayleigh-Bénard convection. After the start of a heat current at constant pressure, an overshoot in $\Delta T(t)$ is routinely observed in Boussinesq fluids. The origin of the overshoot is a certain "inertia" of the fluid immediately after the heat flux is applied: Even though the final state is convection, initially the fluid remains stationary and $\Delta T(t)$ follows the solution of the thermal diffusion equation. As the fluid begins to move, the trace shows an overshoot with a peak at $t_{\mathrm{p}}$ followed by a transition to the steady state value in the convective regime as described, for example, by Behringer [13]. Thus, $t_{p}$ approximately describes the time that it takes for the layer to develop convective motion.

In a compressible fluid, the non-convecting state during the initial transient is expected to persist up to a value

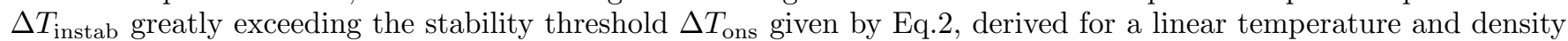
distribution in the vertical direction. This result for $\Delta T_{\text {instab }}$ was obtained by El Khouri and Carlès [14] via a linear stability analysis and is a direct consequence of the strongly inhomogeneous vertical density and temperature distribution with pronounced boundary layers generated by the Piston effect [15]. A clear example can be seen in Fig. 1(a) of ref. [4], which presents transient curves for $\epsilon=0.05$. The simulated trace essentially coincides with the 
zero-gravity result (Eq.3.3 in ref [4]) up to $\Delta T(t)$ of order $280 \mu \mathrm{K}$, while the stability criterion (Eq.2 in the present paper) gives only $7 \mu \mathrm{K}$. Another example is obtained by comparing the simulations in Fig.2 of ref [5] at $\epsilon=0.01$ with calculations under zero gravity, which also shows the two curves coinciding until close to $t_{\mathrm{p}}$. Hence the simulations $[4,5]$ are qualitatively consistent with the predictions of linear stability analysis.

We now recapitulate the main findings of the comparison between experimental data and simulations for a supercritical very compressible fluid, ${ }^{3} \mathrm{He}$. Starting with $t_{\mathrm{osc}}$, which is the characteristic time determined farthest away from the start of the heat flow, both experiments and simulations in the scaled representation are in good agreement. By contrast, as shown in Fig.5, $t_{\mathrm{p}}$ is systematically larger in the simulations than in the experiments, the difference becoming more important as $\epsilon$ increases. An intriguing puzzle is why all the experimental $t_{\mathrm{p}}$ data at the various values of $\epsilon$ can be cast into a scaling representation, while the simulation data cannot.

This disagreement for $t_{\mathrm{p}}$ is surprising: at large $\epsilon$ where the compressibility of the fluid has become smaller, a "simple" Boussinesq behavior should be recovered. A possible origin of this discrepancy is the imperfection of the temperature control of the top plate during the experiment. The signal from the control thermometer [1] is recorded by a circuit with a time constant of 1.25 seconds and $6 \mathrm{~dB} /$ octave rolloff. Therefore, temperature fluctuations with frequencies above a few $\mathrm{Hz}$ cannot be detected. The average temperature noise is estimated to be about $1 \mu K / \sqrt{H z}$ r.m.s. Because of very high thermal conductivity of the top plate material (OFHC copper) it seems reasonable to assume that this perturbation produces no horizontal temperature gradients. It is therefore possible that small parasitic fluctuations of the top plate temperature could speed up the development of the convecting state, hence producing an overshoot with a smaller $t_{\mathrm{p}}$ than the simulations do, which have no noise. We would anticipate that once the convection is almost fully developed, the influence of the fluctuations mentioned above would not be noticeable any more. Hence they might not affect the period $t_{\text {osc }}$ of the damped oscillations, which would explain the good agreement between experiment and simulations and also that in the steady-state condition [16].

We mention here that in the experiments [1] a rounding of the onset point on the steady-state $\Delta T(q)$ measurements was reported and also attributed to the top plate noise. The rounding was found to become more and more pronounced as the critical point point was approached, i.e. the effect of the fluctuations would have to be increasing with decreasing $\epsilon$. The effect of time-dependent boundary conditions on convection in a compressible fluid appears to be an interesting and open question. We suggest future studies, both experimental and numerical ones, that would focus on the transient response of a fluid layern a R-B cell to an externally imposed perturbation of the top surface temperature.

\section{CONCLUSIONS}

A systematic comparison of the results from experimental and 2D simulation convection studies of a compressible fluid has been reported. This fluid is supercritical $\mathrm{He}^{3}$ along the critical isochore and over a temperature range where the compressibility varies by a factor of $\approx 40$. The temperature profile $\Delta T(t)$ across the fluid layer in a Rayleigh-Bénard cell after the start of a heat flow was investigated in both experiments and simulations. The damped oscillations in the transient after the start of the heat flow, and with a period $t_{\text {osc }}$ are of particuliar interest. They are a consequence of vertical mass flows that result from the "piston effect" triggered by the plumes - both moving up and down.

The comparison of the steady-state results, expressed in terms of the convection current versus the Rayleigh number, shows good agreement in general. However, the comparison of the transient results shows some systematic discrepancies, which appear to become more important as the compressibility decreases (i.e as the distance from the critical point increases). This can be seen clearly by examining the respective $\Delta T(t)$ profiles at various temperatures. The agreement is best for the oscillation period where the simulation results and the experimental data can be represented in scaled form versus the Rayleigh number. The absence of noise in the simulations, in contrast to a physical system, might be a possibility for the discrepancy. In addition, as mentioned earlier in this paper, the simulations carried out independently by two research groups $[4,5]$ do not detect the slow relaxation of $\Delta T(t)$ to the steady state, or the region of "truncated oscillations" observed in the experiments. These discrepancies remain to be understood.

\section{ACKNOWLEDGMENTS}

The authors thank Fang Zhong for his efficient help with the formatting of several figures in this paper and the fitting of data sets. One of the authors (HM) is greatly indebted to S. Amiroudine and to P. Carlès for correspondance and discussions. The helpful comments of R. P. Behringer and P. Carlès on the manuscript have been greatly appreciated. The work is supported by the NASA grant NAG3-1838 and by the Japan Space forum H12-264. 


\section{APPENDIX}

\section{A. Boundary between regimes with damped and "truncated" oscillations.}

It is useful to present this boundary in terms of the Rayleigh number difference $\left[R a_{\text {corr }}^{\text {bound }}-R a_{c}\right]$ versus $\epsilon$, where the transformation from the former representation $\Delta T_{\text {bound }}(\epsilon)$ to the present one was done via Eq.6 of ref. [2]. The zone diagram in this format shows that the boundary height remains constant at $\left[R a_{\text {corr }}^{\text {bound }}-R a_{c}\right]=700 \pm 200$ for $0.05<\epsilon<0.2$, and then rises steeply as $\epsilon$ further decreases. This is shown in Fig.7.

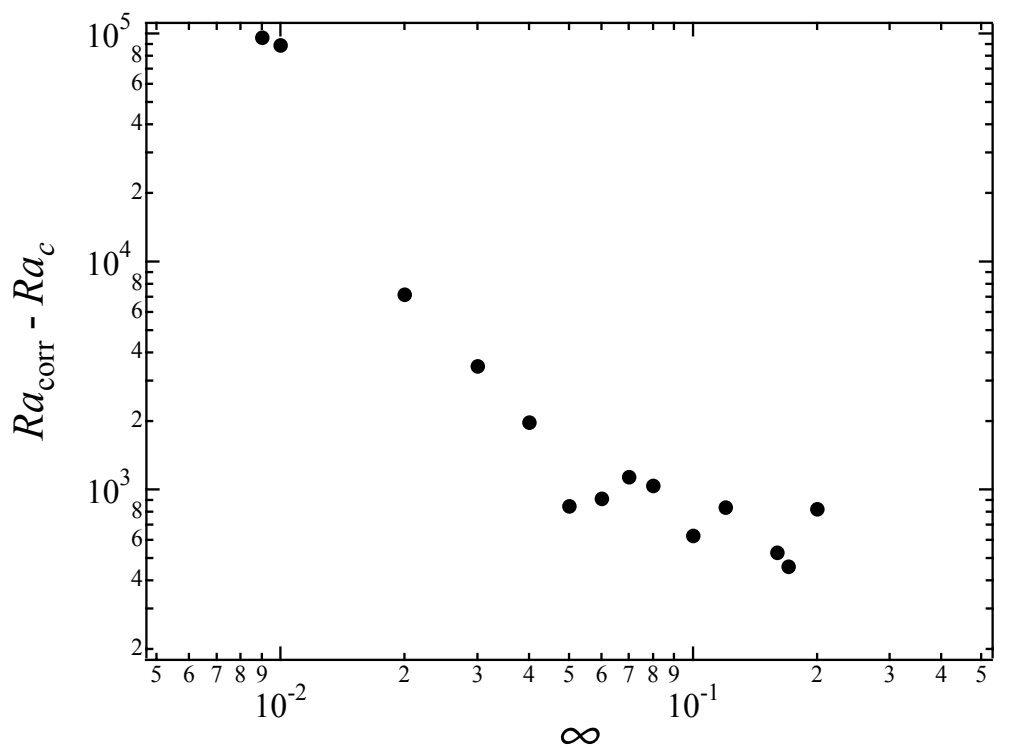

FIG. 6. The boundary between the regimes showing damped oscillations (top region) and truncated oscillations (bottom region), expressed in terms of $\left[R a_{\text {corr }}-R a_{c}\right]$ versus $\epsilon$.

\section{B. The time $t_{\mathrm{m}}$ in the profile $\Delta T(t)$}

The discussion of $t_{\mathrm{m}}$, the time defined in Fig.2, is more complex than for $t_{\mathrm{osc}}$ and $t_{\mathrm{p}}$. In Fig. 7 , both the results for $t_{\mathrm{m}} / \tau_{D}$ versus $\left[R a_{\text {corr }}-R a_{c}\right]$ from the analysis of the experimental and the simulated transient profiles $\Delta T(t)$ are presented. Both sets of results are clearly separated by a roughly constant amount on the vertical logarithmic scale. Hence we can use the same symbols for the values of $\epsilon$ for the experiments and the simulation. For the sake of simplicity, we do not distinguish between the various authors in this figure. We note, however,that the simulation points for $\left[R a_{c o r r}-R a_{c}\right]=5.96 \times 10^{4}$ and $4.78 \times 10^{5}$ at $\epsilon=0.01$ are those by Amiroudine [5]. Here, in contrast with $t_{\text {osc }}$, the $t_{m}$ data from experiments show a small but systematic departure from collapsing on a single curve at the lower values of $\left[R a_{c o r r}-R a_{c}\right]$. However the scaled results from simulations at the various values of $\epsilon$ collapse on one curve within the scatter. The trend is the same for both experimental and simulation data : the limiting slopes of the curves at the extremities of $\left[R a_{c o r r}-R a_{c}\right]$ correspond to power laws with exponents of approximately $\mathrm{n}=-1$ and $\mathrm{n}=-0.5$, respectively, at the low - and at the high end of the $R a$ numbers. This is shown in Fig.7.

We note that 1 ) the experimental data points for $\left[R a_{\text {corr }}-R a_{c}\right]$ below the values of the boundary shown in Fig. 6 are in the regime of "truncated" oscillations. They form a smooth continuation of the curves at higher values of $\left[R a_{\text {corr }}-R a_{c}\right]$, namely in the regime of damped oscillations. 2) The experimental data for $\epsilon \geq 0.07$, which collapse on a single curve are those where the boundary in Fig. 6 is at $\left[R a_{\text {corr }}-R a_{c}\right] \approx$ const $=700$. The departure from collapse from the first onto a second curve takes place when the boundary in Fig.6 begins to rise steeply for $\epsilon \leq 0.07$. 3) It is intriguing that the logarithmic vertical separation of the experimental and simulation curves corresponds to roughly a factor of 2 . This means that for the same value of $\left[R a_{c o r r}-R a_{c}\right]$, the observed $t_{\mathrm{m}}$ has roughly twice the value of $t_{\mathrm{m}}$ from simulations.

The analysis of the experimental $t_{m}$ data for the limit of low values of $\left[R a_{c o r r}-R a_{c}\right]$ and the comparison with a simple predicted expression by Chiwata and Onuki [3] was already presented in Figs. 7 and 8 of ref. [2]. The exponent of -1 found both for the experiments and the simulations is consistent with the simple prediction. 


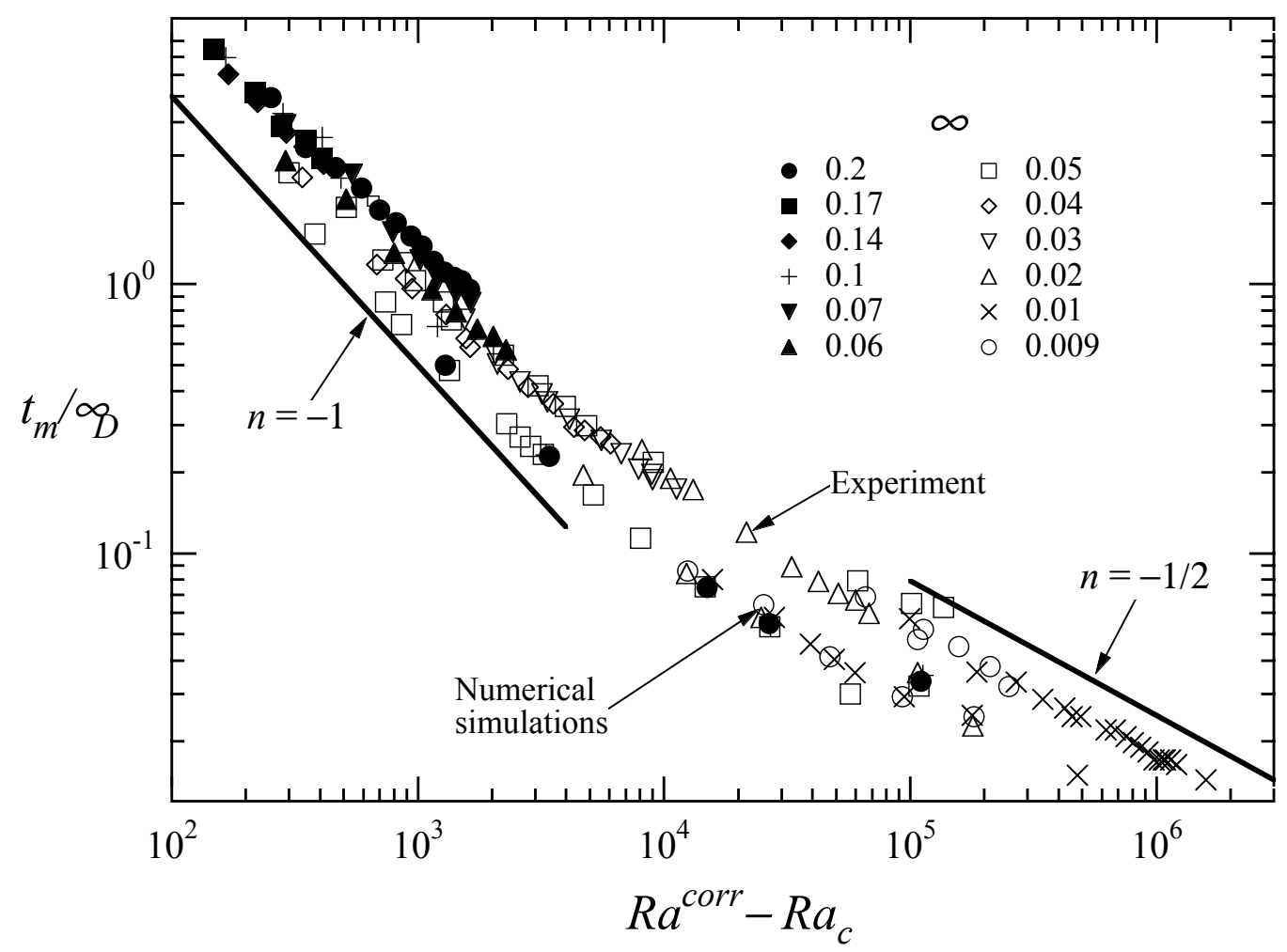

FIG. 7. The time $t_{\mathrm{m}}$ between the first peak and the first minimum, scaled by the diffusion time $\tau_{D}$, versus $\left[R a_{\text {corr }}-R a_{c}\right]$. The experimental data are located on the upper two almost overlapping trajectories of symbols. Those from the simulation, showing a collapse, are located on the lower trajectory of symbols. The symbols denote the various values of $\epsilon$ at which both experiments and simulations were carried out.

[1] A.B. Kogan and H. Meyer, Phys. Rev. E 63, 056310 (2001).

[2] H. Meyer and A.B. Kogan, Phys. Rev. E 66,056310 (2002).

[3] Y. Chiwata and A. Onuki, Phys. Rev. Lett. 87, 144301 (2001).

[4] A. Furukawa and A. Onuki Phys. Rev. E 66, 016302 (2002).

[5] S. Amiroudine and B. Zappoli, Phys. Rev. Lett. 90, 105303 (2003), and S. Amiroudine (private communication).

[6] L.D. Landau and E.M. Lifshitz, Course of Theoretical Physics: Vol.6 Fluid Mechanics (Pergamon, Oxford, 1959)

[7] G. Ahlers, M. Cross, P. Hohenberg and S. Safran. J. Fluid Mech.110, 297 (1982).

[8] M. Gitterman and V. Steinberg, J. Appl. Math. Mech. USSR 34, 305 (1971). M. Gitterman, Rev. Mod. Phys. 50, 85 (1978).

[9] P. Carlès and B. Ugurtas, Physica D 126, 69 (1999).

[10] D.J. Tritton, Physical Fluid Dynamics (Oxford Science, Oxford, 1988), Section 14.6.

[11] A. Schlueter, D. Lortz and F. Busse, J. Fluid Mech. 23, 129 (1965).

[12] R.P. Behringer, A. Onuki and H. Meyer, J. Low Temp. Phys.81, 71, (1990).

[13] R.P. Behringer, Rev. Mod. Phys. 57, 657 (1985).

[14] L. El Khouri and P. Carlès, Phys. Rev. E 66, 066309 (2002).

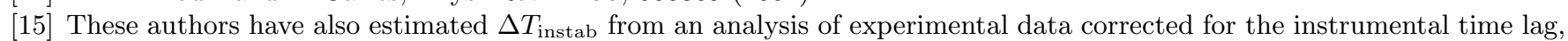
and have found them to be consistent with their calculations for scenarios (a) and (b) where the initial rise of $\Delta T(t)$ is not too rapid and the correction can be carried out (L. El Khouri and P. Carlès, to be published, private communication).

[16] The absence of noise in simulations, its presence in a physical system, and the resulting impact on the growth of perturbations in the convective state was pointed out to one of us (HM) by P. Carlès (private communication). 